149:1788-1794.

8. Dickinson, A.M., et al. 2002. In situ dissection of the graft-versus-host activities of cytotoxic T cells specific for minor histocompatibility antigens. Nat Med. 8:410-414.

9. van der Harst, D., et al. 1994. Recognition of minor histocompatibility antigens on lymphocytic and myeloid leukemic cells by cytotoxic T-cell clones. Blood. 83:1060-1066.

10. Marijt, W.A., et al. 2003. Hematopoiesis-restricted minor histocompatibility antigens HA-1- or HA-2 specific $\mathrm{T}$ cells can induce complete remissions of relapsed leukemia. Proc. Natl. Acad. Sci. U. S. A. 100:2742-2747.

11. Goulmy, E. 2004. Minor histocompatibility antigens: allo target molecules for tumor-specific immunotherapy. Cancer J. 10:1-7.

12. Mutis, T., et al. 1999. Feasibility of immunotherapy of relapsed leukemia with ex vivo- generated cytotoxic T lymphocytes specific for hematopoietic system-restricted minor histocompatibility antigens. Blood. 93:2336-2341.

13. Spierings, E., Wieles, B., and Goulmy, E. 2004 Minor histocompatibility antigens - big in tumour therapy. Trends Immunol. 25:56-60.

14. Rotzschke, O., Falk, K., Wallny, H.J., Faath, S., and Rammensee, H.G. 1990. Characterization of naturally occurring minor histocompatibility peptides including H-4 and H-Y. Science. 249:283-287.

15. den Haan, J.M., et al. 1995. Identification of a graft versus host disease-associated human minor histocompatibility antigen. Science. 268:1476-1480.

16. Dolstra, H., et al. 1999. A human minor histocompatibility antigen specific for B cell acute lymphoblastic leukemia. J. Exp. Med. 189:301-308.

17. Murata, M., Warren, E.H., and Riddell, S.R. 2003. A human minor histocompatibility antigen resulting from differential expression due to a gene deletion. J. Exp. Med. 197:1279-1289.

18. Vogt, M.H., de Paus, R.A., Voogt, P.J., Willemze, R., and Falkenburg, J.H. 2000. DFFRY codes for a new human male-specific minor transplantation antigen involved in bone marrow graft rejection. Blood. 95:1100-1105

19. Vogt, M.H., et al. 2000. UTY gene codes for an HLA-B60-restricted human male-specific minor histocompatibility antigen involved in stem cell graft rejection: characterization of the critical polymorphic amino acid residues for $\mathrm{T}$-cell recognition. Blood. 96:3126-3132.

20. Vogt, M.H., et al. 2002. The DBY gene codes for an HLA-DQ5 restricted human male specific minor histocompatibility antigen involved in GvHD. Blood. 99:3027-3032.

21. Spierings, E., et al. 2003. Identification of HLA class II-restricted H-Y-specific T-helper epitope evoking CD4+ T-helper cells in H-Y-mismatched transplantation. Lancet. 362:610-615.

22. Gubarev, M.I., et al. 1996. Localization to chromosome 22 of a gene encoding a human minor histo- compatibility antigen. J. Immunol. 157:5448-5454. 23. Dausset, J., et al. 1990. Centre d'etude du polymorphisme humain $(\mathrm{CEPH})$ : collaborative genetic mapping of the human genome. Genomics. 6:575-577.

24. Murray, J.C., et al. 1994. A comprehensive human linkage map with centimorgan density. Cooperative Human Linkage Center (CHLC). Science. 265:2049-2054.

25. Gubarev, M.I., et al. 1998. Localization to chromosome 11 of a gene encoding a human minor histocompatibility antigen. Exp. Hematol. 26:976-981.

26. Warren, E.H., et al. 2002. Feasibility of using genetic linkage analysis to identify the genes encoding $\mathrm{T}$ cell-defined minor histocompatibility antigens. Tissue Antigens. 59:293-303.

27. Akatsuka, Y., et al. 2003. Identification of a polymorphic gene, BCL2A1, encoding two novel hematopoietic lineage-specific minor histocompatibility antigens. J. Exp. Med. 197:1489-1500.

28. de Rijke, B., et al. 2005. A frameshift polymorphism in $P 2 X 5$ elicits an allogeneic cytotoxic T lymphocyte response associated with remission of chronic myeloid leukemia. J. Clin. Invest. 115:3506-3516. doi:10.1172/JCI24832.

29. den Haan, J.M., et al. 1998. The minor histocompatibility antigen HA-1: a diallelic gene with a single amino acid polymorphism. Science. 279:1054-1057.

30. Warren, E.H., et al. 2000. The human UTY gene encodes a novel HLA-B8-restricted H-Y antigen. J. Immunol. 164:2807-2814.

\title{
Flushing out the role of GPR109A (HM74A) in the clinical efficacy of nicotinic acid
}

\author{
Nicholas B. Pike
}

Atherosclerosis Department, GlaxoSmithKline, Stevenage, United Kingdom.

\begin{abstract}
The recent discovery of the $G_{i}$ protein-coupled receptor GPR109A (HM74A in humans; PUMA-G in mice) as a receptor for nicotinic acid has provided the opportunity to gain greater understanding of the underlying biology contributing to the clinical efficacy (increases in HDL, decreases in VLDL, LDL, and triglycerides) and the characteristic side-effect profile of nicotinic acid. GPR109A has been proven to be the molecular target for the actions of nicotinic acid on adipose tissue, and in this issue of the JCI, Benyó et al. have confirmed the involvement of GPR109A in the nicotinic acid-induced flushing response, a common side effect (see the related article beginning on page 3634). The involvement of GPR109A in both the desirable and undesirable clinical actions of nicotinic acid raises interesting questions regarding the function of this receptor.
\end{abstract}

The observation that nicotinic acid can modify lipoprotein profiles in humans was first made in the 1950s. Subsequent clinical experience has demonstrated that nicotinic acid produces a very beneficial modification of multiple cardiovascular risk factors. As a

Nonstandard abbreviations used: $\mathrm{PGD}_{2}$, prostaglandin $\mathrm{D}_{2} ; \mathrm{PGE}_{2}$, prostaglandin $\mathrm{E}_{2} ;$ PUMA-G, protein upregulated in macrophages by IFN- $\gamma$.

Conflict of interest: The author has declared that no conflict of interest exists.

Citation for this article: J. Clin. Invest. 115:3400-3403 (2005). doi:10.1172/JCI27160. monotherapy, nicotinic acid is still the most effective therapy for elevating HDL levels while decreasing VLDL and LDL levels as well as other cardiovascular risk factors, which results in a reduction in mortality (1) (Figure 1). In addition to its highly desirable profile of cardiovascular risk factor modulation, nicotinic acid has been shown to produce enhanced therapeutic effects when given in combination with other lipid-lowering drugs (e.g., statins and bile acid resins) (2-3). The past 50 years of nicotinic acid usage has been recently reviewed by Carlson (4).

\section{Identification and function of $G_{\text {i }}$ protein-coupled receptors for nicotinic acid}

In 2003, several groups published studies showing that the orphan receptor GPR109A is activated by nicotinic acid at concentrations consistent with the exposure achieved following therapeutic doses (5-7). Furthermore, additional compounds with a clinical profile similar to that of nicotinic acid (e.g., acipimox and acifran) were also confirmed as full agonists of GPR109A. Importantly, nicotinamide, which does not alter lipoprotein profiles but shares the vitamin-like properties of nicotinic acid, has virtually no GPR109A agonist activity. This pharmacological profile strongly suggests that GPR109A is a molecular target involved in the clinical efficacy of nicotinic acid and therefore offers a potential focus to explore the biological processes involved in the highly desirable therapeutic profile achieved following chronic treatment with this drug (8-9).

The best-described action of nicotinic acid is the inhibition of adipocyte lipolysis. 

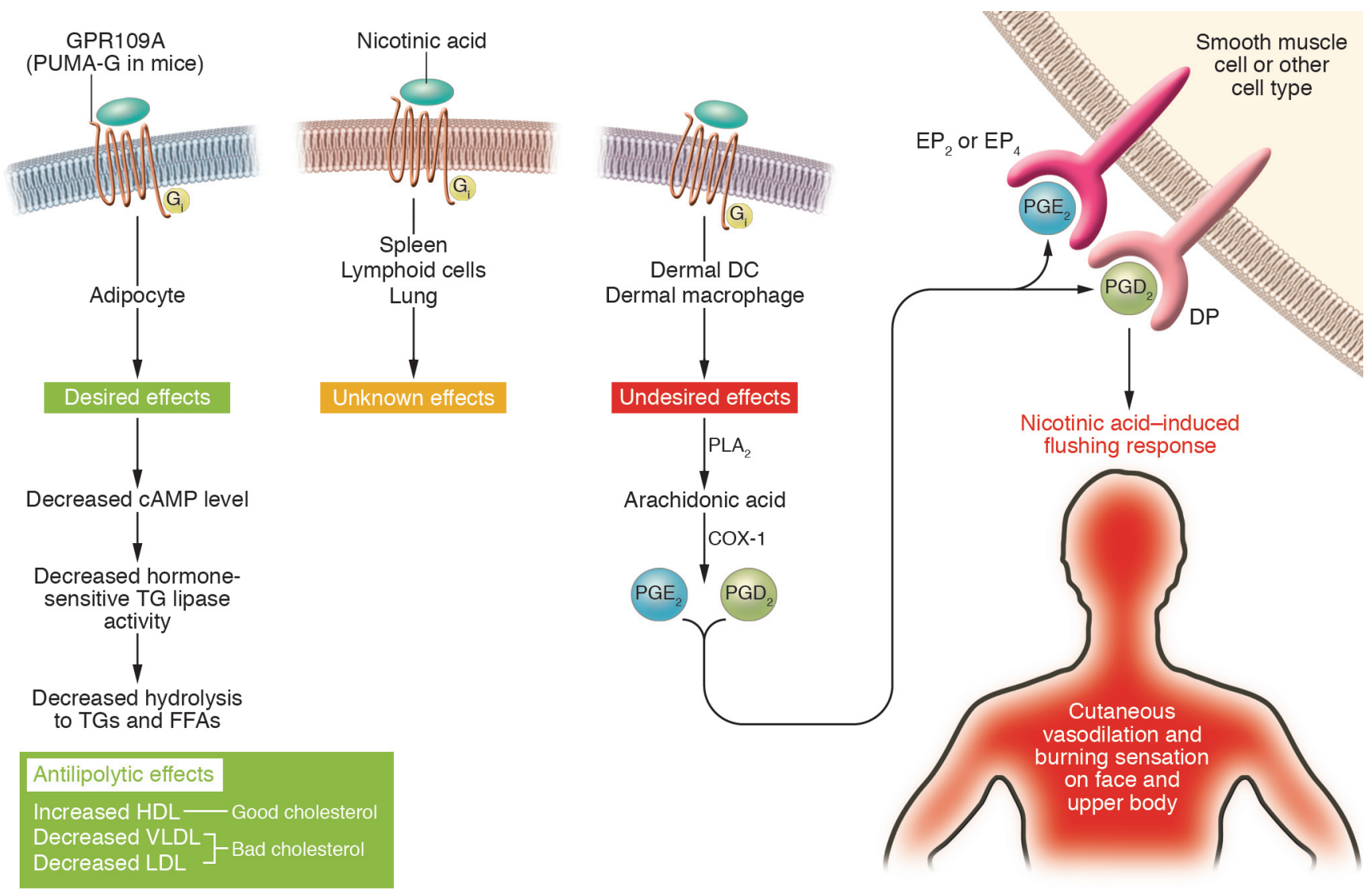

Figure 1

Activation of the $G_{i}$ protein-coupled receptor GPR109A (HM74A in humans; PUMA-G in mice) can produce differential responses depending on the location of the receptor. It has been proposed that, when nicotinic acid activates GPR109A on adipocytes, the resultant antilipolytic effects contribute to the highly desirable normalization of lipoprotein profiles. However, when nicotinic acid activates GPR109A on dermal dendritic cells or dermal macrophages, the subsequent mobilization of arachidonic acid and its conversion to vasodilatory prostaglandins $\left(\mathrm{PGD}_{2}\right.$ and $\mathrm{PGE}_{2}$ ) results in the characteristic flushing response. As GPR109A expression extends beyond adipose and immune cells located in the skin (e.g., spleen, lymphoid cells, and lung), it is likely that activation of GPR109A in these cells/tissues may also contribute to the clinical efficacy of nicotinic acid. PLA 2 , phospholipase $\mathrm{A}_{2}$; TG, triglyceride.

The combination of pharmacology and the use of transgenic mice has confirmed that GPR109A expressed in adipocytes is functionally competent (5-7). A key question is whether the activity of nicotinic acid via GPR109A expressed on the surface of the adipocyte is sufficient to account for the overall clinical efficacy observed. The lowering of circulating nonesterified fatty acid levels via activation of adipocyte GPR109A would be expected to lead to a reduction in hepatic triglyceride synthesis and hence circulating VLDL levels. Due to the inverse relationship between plasma triglycerides and HDL cholesterol, it is likely that HDL levels would be increased if the reduction in VLDL resulted in an inhibition of cholesterol ester transfer protein activity (10).

In addition to expression on adipocytes, GPR109A is known to be most highly expressed in spleen, lung, and lymphoid cells (5). However, the lack of good-quality antibodies has failed to determine whether mRNA expression is always accompanied by functional levels of GPR109A protein. To date, there is very little evidence in the literature for GPR109A-mediated effects outside of adipose tissue. The obvious exception to this is the intense cutaneous vasodilation (flushing) that has been seen clinically with each GPR109A agonist tested to date. In this issue of the JCI, Benyó et al. show that protein upregulated in macrophages by IFN- $\gamma$ (PUMA-G), the murine ortholog of GPR109A, is responsible for the nicotinic acid-induced flushing response (11). In their murine studies, this group has demonstrated a GPR109A-mediated mobilization of arachidonic acid, which is converted to prostaglandins to result in the characteristic vasodilatory response (Figure 1). This elegant study confirms the proposed mechanism from clinical observation (12) and supports the hypothesis that immune cells in the skin are the most likely source of arachidonic acid and prostaglandins. Clearly, in the mouse, both $\mathrm{PGE}_{2}$, via the type 2 and type $4 \mathrm{PGE}_{2}$ receptors $\left(\mathrm{EP}_{2}\right.$ and $E_{4}$, respectively), and $\mathrm{PGD}_{2}$, via the $\mathrm{PGD}_{2}$ receptor (DP), are involved in nicotinic acidinduced cutaneous vasodilation. However, in humans, the precise involvement of the prostanoid receptor is yet to be resolved, but based on the analysis of metabolites, DP is likely to be involved (12).

The confirmation that the nicotinic acidinduced flushing response is GPR109A mediated raises several interesting questions. First, will it be possible to produce GPR109A agonists with the beneficial efficacy of nicotinic acid but without the marked flushing response associated with nicotinic acid? Alternative formulations, 
prodrugs, and different GPR109A ligands (e.g., acipimox and acifran) have been claimed to induce less flushing than crystalline nicotinic acid $(13,14)$. Therefore, as the chemical diversity of future GPR109A ligands is increased, it is conceivable that compounds that do not compartmentalize into the skin could induce markedly less flushing than that induced by nicotinic acid. Second, the involvement of GPR109A in the flushing response raises the question of whether GPR109A receptors located outside adipose tissue can contribute to the clinical efficacy of nicotinic acid. GPR109A is highly expressed in lymphoid cells. This expression profile, coupled with the observation that in murine macrophages, GPR109A is upregulated by cytokines like IFN- $\gamma(15)$, may suggest a role for GPR109A in immunity and inflammation. If this is the case, then it is interesting to speculate on whether nicotinic acid achieves any of its clinical efficacy via these cell types (e.g., at the level of the atherosclerotic plaque) and not solely via the normalization of aberrant lipoprotein profiles. Furthermore, one could speculate on a degree of analogy with the mechanism of action that results in the flushing response, for example, the generation of prostaglandins following the liberation of archidonic acid via GPR109A stimulation. If this functionality of GPR109A is indeed involved in the clinical efficacy of nicotinic acid, it raises further areas for investigation, which would include determination of whether the tachyphylaxis observed in the flushing response to nicotinic acid and acipimox is observed in other immune/ inflammatory cell types and whether the symptomatic relief from flushing achieved by NSAID administration has detrimental effects on some aspects of the efficacy of GPR109A agonists. It is apparent that the lipoprotein changes achieved with nicotinic acid are not affected by NSAID treatment (16), possibly suggesting the involvement of a direct GPR109A-mediated event.

\section{Endogenous ligand for GPR109A and related receptors}

The general consensus in the literature is that nicotinic acid is not the endogenous ligand for GPR109A $(4,8,9)$. This consensus is primarily based on the fact that physiological levels of nicotinic acid are insufficient to activate GPR109A. It has been suggested that D- $\beta$-hydroxybutyrate, a fatty acid-derived ketone body, is an endogenous ligand for GPR109A (17). Although D- $\beta$-hydroxybutyrate is approxi- mately 8000 times less active than nicotinic acid on GPR109A (or PUMA-G), it can reach concentrations sufficient to activate this receptor under certain conditions (e.g., starvation). It has therefore been proposed that $D$ - $\beta$-hydroxybutyrate is involved in a homeostatic mechanism for starvation survival that involves regulating its own production, preventing ketoacidosis, and regulating fat utilization (17).

GPR109A shares a high degree of homology with 2 additional receptors found on chromosome 12q24.31 (5). GPR109B (HM74 in humans) shares $96 \%$ homology with GPR109A while GPR81 shares $60 \%$ homology. Neither nicotinic acid nor D- $\beta$-hydroxybutyrate are potent agonists of GPR81 or GPR109B, and only nicotinic acid exhibits any activity. Nicotinic acid has been shown to have an agonistic effect on GPR109B in the $\mathrm{mM}$ range, which is approximately 1,000 fold weaker than its effect on GPR109A (5). Acifran, in contrast, is only 10-fold weaker at GPR109B than at GPR109A, indicating that a single molecule can exhibit similar activity on both GPR109A and GPR109B (5). It is interesting to speculate that a single molecule or a group of closely related molecules may activate all 3 of these human receptors.

\section{Alternative mechanisms for nicotinic acid efficacy}

Alternative mechanisms, likely to be independent of GPR109A activation, have been proposed for the lipoprotein-modulating actions of nicotinic acid. Application of high concentrations ( $\mathrm{mM}$ ) of nicotinic acid inhibits hepatocyte diacylglycerol acyltransferase and triglyceride synthesis, leading to increased intracellular apoB degradation in HepG2 cells (18). Nicotinic acid also inhibits the uptake and removal of HDLApoAI (but not HDL-cholesterol ester), thereby resulting in increased HDL-ApoAI levels in the media of cultured HepG 2 cells (19). This work has been recently reviewed (20), and the responses to nicotinic acid are unlikely to be GPR109A-mediated, as receptor expression has not been detected in hepatocytes. Concentrations of nicotinic acid in the $\mathrm{mM}$ range can stimulate both CD36 and ATP-binding cassette A1 (ABCA1) in cultured monocytoid cells (21), leading to enhancement of cholesterol removal and of HDL-mediated cholesterol efflux. Furthermore, it is unlikely that these effects are GPR109A mediated, based on the pharmacology presented, with aspirin inhibiting the observed upregulation of CD36 but not ABCA1 and protein kinase A inhibition blocking ABCA1 upregulation but not CD36. In both of these cases, it would be interesting to test alternative GPR109A agonists as well as nicotinamide in order to put these observations into context with observed clinical responses.

To conclude, GPR109A represents an exciting molecular target. Further examination of this receptor may facilitate our understanding of the mechanism of action of nicotinic acid and thereby aid the development of improved therapeutic options for the treatment of cardiovascular disease. Utilizing the tissue distribution profile of GPR109A and the increasing number of agonist molecules available as well as developing studies using nicotinamide as a negative control, we may finally resolve the elusive mechanism of action for nicotinic acid.

Address correspondence to: Nicholas B. Pike, Atherosclerosis Department, GlaxoSmithKline, Gunnels Wood Road, Stevenage, Hertfordshire, SG1 2NY, United Kingdom. Phone: 44-01438-764178; Fax: 44-01438763232; E-mail: Nick.B.Pike@gsk.com.

1. Tavintharan, S., and Kashyap, M.L. 2001. The benefits of niacin in atherosclerosis. Curr. Atheroscler. Rep. 3:74-82.

2. Moon, Y.S., and Kashyap, M.L. 2002. Niacin extended-release/lovastatin: combination therapy for lipid disorders. Expert Opin. Pharmacother. 12:1763-1771.

3. Crouse, J.R. 1996. New developments in the use of niacin for treatment of hyperlipidemia: new considerations in the use of an old drug. Coron. Artery Dis. 7:321-326.

4. Carlson, L.A. 2005. Nicotinic acid: the broad-spectrum lipid drug. A 50th anniversary review. J. Intern. Med. 258:94-114.

5. Wise, A., et al. 2003. Molecular identification of high and low affinity receptors for nicotinic acid. J. Biol. Chem. 278:9869-9874.

6. Soga, T., et al. 2003. Molecular identification of nicotinic acid receptor. Biochem. Biophys. Res. Commun. 303:364-369.

7. Tunaru, S., et al. 2003. PUMA-G and HM74 are receptors for nicotinic acid and mediate its antilipolytic effect. Nat. Med. 9:352-355.

8. Pike, N.B., and Wise, A. 2004. Identification of a nicotinic acid receptor: is this the molecular target for the oldest lipid-lowering drug? Curr. Opin. Invertig. Drugs. 5:271-275.

9. Karpe, F., and Frayn, K.N. 2004. The nicotinic acid receptor - a new mechanism for a new drug. Lancet. 363:1892-1894.

10. Mann, C.J., Yen, F.T., Grant, A.M., and Bihain, B.E. 1991. Mechanism of plasma cholesteryl ester transfer in hypertriglyceridemia. J. Clin. Invest. 88:2059-2066.

11. Benyó, Z., et al. 2005. GPR109A (PUMA-G/HM74A) mediates nicotinic acid-induced flushing. J. Clin. Invest. 115:3634-3640. doi:10.1172/JCI23626.

12. Morrow, J.D., Awad, J.A., Oates, J.A., and Roberts, L.J., 2nd. 1992. Identification of skin as a major site of prostaglandin D2 release following oral administration of niacin in humans. J. Invest. Dermatol. 98:812-815.

13. Guyton, J.R. 2004. Extended-release niacin for modifying the lipoprotein profile. Expert Opin. 
Pharmacother. 5:1385-1398.

14. Tornvall, P., and Walldius, G. 1991. A comparison between nicotinic acid and acipimox in hypertriglyceridaemia - effects on serum lipids, lipoproteins, glucose tolerance and tolerability. J. Intern. Med. 230:415-421.

15. Schaub, A., Futterer, A., and Pfeffer, K. 2001. PUMA-G, an IFN-gamma-inducible gene in macrophages is a novel member of the seven transmembrane spanning receptor superfamily. Eur. J. Immunol. 31:3714-3725.

16. Carlson, L.A. 1978. Nicotinic acid and inhibition of fat mobilizing lipolysis. Present status of effects on lipid metabolism. Adv. Exp. Med. Biol. 109:225-238.

17. Taggart, A.K.P., et al. 2005. (D)- $\beta$-Hydroxybutyrate inhibits adipocyte lipolysis via the nicotinic acid receptor PUMA-G. J. Biol. Chem. 280:26649-26652.

18. Jin, F.Y., Kamanna, V.S., and Kashyap, M.L. 1999. Niacin accelerates intracellular ApoB degradation by inhibiting triacylglycerol synthesis in human hepatoblastoma (HepG2) cells. Arterioscler. Thromb. Vasc. Biol. 19:1051-1059.

19. Jin, F.Y., Kamanna, V.S., and Kashyap, M.L. 1997. Niacin decreases the removal of high-density lipo- protein apolipoprotein A-I but not cholesterol ester by HepG2 cells. Implications for reverse cholesterol transport. Arterioscler. Thromb. Vasc. Biol. 17:2020-2028.

20. Ganji, S.H., Kamanna, V.S., and Kashyap, M.L. 2003. Niacin and cholesterol: role in cardiovascular disease. J. Nutr. Biochem. 14:298-305.

21. Rubric, T., Trottmann, M., and Lorenz, R.L. 2004. Stimulation of CD36 and the key effector of reverse cholesterol transport ATP-binding cassette A1 in monocytoid cells by niacin. Biochem. Pharmacol. 67:411-419.

\title{
Desperately seeking sugar: glial cells as hypoglycemia sensors
}

\author{
Amira Klip ${ }^{1}$ and Meredith Hawkins ${ }^{2}$ \\ ${ }^{1}$ Cell Biology Programme, The Hospital for Sick Children, Toronto, Ontario, Canada. 2Division of Endocrinology, Department of Medicine,
} Albert Einstein College of Medicine of Yeshiva University, New York, New York, USA.

\begin{abstract}
A life-saving response to hypoglycemia requires rapid sensing of decreases in glycemia and consequent brisk glucagon secretion. Preceding studies have shown that mice lacking glucose transporter type 2 (GLUT2) lose this response. In this issue of the JCI, Marty et al. report that glucose sensing and consequent pancreatic glucagon secretion are restored by re-expression of GLUT2 in glial but not neuronal cells (see the related article beginning on page 3545). A new, glucose-sensing role is ascribed to GLUT2expressing glial cells.
\end{abstract}

\section{Central responses to hypoglycemia}

Hypoglycemia is a life-threatening condition in which blood glucose can drop to levels that jeopardize brain function. Insulin and antihyperglycemic therapies are major causes of dangerous hypoglycemic episodes in diabetic individuals. With the global prevalence of the disease and ambitious goals for blood sugar reduction, hypoglycemia has become a clinically significant health issue. The ability to rapidly sense a drop in circulating glucose and induce a counterregulatory response forms the cornerstone of the body's life-saving responses to hypoglycemia. Accordingly, several systems participate in glucose sensing and the evoked secretion of glucagon and adrenalin. How is hypoglycemia detected in the first place? The following have been proposed: (a) glucose levels may be sensed by the glucagon-secreting, pancreatic $\alpha$ cells; (b) low intrapancreatic insulin levels may regulate glucagon secretion; and (c) the

Nonstandard abbreviations used: GLUT2, glucose transporter type 2; NTS, nucleus of the tractus solitarius.

Conflict of interest: The authors have declared that no conflict of interest exists.

Citation for this article: J. Clin. Invest. 115:3403-3405 (2005). doi:10.1172/JCI27208.
CNS may sense the drop in glucose and elicit secretory hormonal responses. Hypoglycemia-evoked glucagon and epinephrine secretion are similarly rapid (within minutes) and activated by comparable blood glucose nadirs (3.6-3.9 mM) (1). However, these hormonal responses may be activated by different mechanisms, each involving the CNS. Glucagon secretion elicited by hypoglycemia is normal in a neuronal insulin-receptor knockout (NIRKO) mouse model, yet the sympathoadrenal response is markedly impaired (2). In contrast, mice lacking ATP-sensitive $\mathrm{K}^{+}$channels display a severe defect in glucagon secretion (despite normal $\alpha$ cell function) yet secrete epinephrine normally in response to hypoglycemia (3). In people with type 1 diabetes, the loss of glucagon secretion with hypoglycemia occurs early in the disease course while the defect in epinephrine secretion becomes manifest later (1).

Injecting glucoprivic agents such as 2-deoxyglucose into either the ventromedial hypothalamus or certain brainstem sites activates neurohumoral counterregulatory responses similar to those seen during systemic hypoglycemia $(4,5)$. Subsequent efferent output to the $\alpha$ cells involves the autonomic nervous system (6). The presence of both glucose transporter type 2 (GLUT2) and glucokinase in some neurons and astrocytes in glucose-sensitive brain regions led to the concept of a central glucose sensor similar to that in pancreatic $\beta$ cells (7). However, although GLUT2 is expressed in up to one-third of glucose-sensing neurons, an equal number of non-glucose-sensing neurons also express this transporter (8). In this issue of the JCI, Marty et al. show that mice lacking GLUT2 are unable to respond to hypoglycemia with increased glucagon secretion despite restoration of glucose-stimulated insulin secretion by $\beta$ cell-selective Glut 1 overexpression (RipGlut1;Glut2-/- mice) (9). Therefore, a picture emerges in which, in addition to hypoglycemia detection by glucose flux into $\alpha$ cells and the intrapancreatic insulin relay, central detection of glucose is paramount and generates an efferent glucagon-releasing response involving glucose transport into glial cells.

\section{Astrocytes/glial cells: first responders to hypoglycemic emergencies?}

At the core of this study (9) is the observation that transgenic overexpression of GLUT2 in glial cells but not neurons restored this vital response in RipGlut1;Glut2-/- mice. Such central detection requires glial cells and likely involves glial cell-neuron communication in central locations, including the nucleus of the tractus solitarius (NTS) and the dorsal motor nucleus of the vagus (Figure 1). 